Trans

continentales
Transcontinentales

Sociétés, idéologies, système mondial

$10 / 11 \mid 2011$

La ruée vers la terre

\title{
Investisseurs versus paysans locaux : vers quelle agriculture irriguée au Mali ?
}

L'exemple du secteur sucrier

Investors vs. local farmers: the future of irrigated agriculture in Mali. The

example of the sugar sector

Amandine Adamczewski, Jean-Yves Jamin et Jean-Philippe Tonneau

\section{OpenEdition}

Journals

Édition électronique

URL : http://journals.openedition.org/transcontinentales/1113

DOI : $10.4000 /$ transcontinentales. 1113

ISBN : 978-2-7351-1572-3

ISSN : 1775-397X

Éditeur

Editions de la maison des sciences de l'homme

Référence électronique

Amandine Adamczewski, Jean-Yves Jamin et Jean-Philippe Tonneau, «Investisseurs versus paysans locaux : vers quelle agriculture irriguée au Mali ? », Transcontinentales [En ligne], 10/11 | 2011,

document 5, mis en ligne le 19 octobre 2011, consulté le 08 septembre 2020. URL : http://

journals.openedition.org/transcontinentales/1113; DOI : https://doi.org/10.4000/transcontinentales.

1113

Ce document a été généré automatiquement le 8 septembre 2020

Tous droits réservés 


\title{
Investisseurs versus paysans locaux : vers quelle agriculture irriguée au Mali ?
}

\author{
L'exemple du secteur sucrier \\ Investors vs. local farmers: the future of irrigated agriculture in Mali. The \\ example of the sugar sector
}

Amandine Adamczewski, Jean-Yves Jamin et Jean-Philippe Tonneau

\section{Introduction}

1 Depuis la crise alimentaire mondiale de 2008, acquérir des terres pour y développer une production agricole est devenu un objectif pour de nombreux pays dépendant des importations alimentaires. Le phénomène des investissements fonciers à grande échelle, qualifié par certains d'accaparements fonciers, s'est amplifié ces dernières années. Le continent africain apparaît comme une cible privilégiée de ces nouveaux investissements ${ }^{1}$. Les motivations des investisseurs sont avant tout d'assurer la sécurité alimentaire de leur pays d'origine en mobilisant les ressources en terre des pays africains. Pour les pays hôtes, le recours à des investisseurs extérieurs doit faciliter la réalisation de leurs propres objectifs de développement : assurer la sécurité alimentaire nationale en modernisant l'agriculture et en intensifiant les productions agricoles. Ces deux objectifs sont-ils compatibles? Nous donnerons ici des éléments de réponse à cette question en nous fondant sur le cas du Mali et de l'office du Niger.

2 Le Mali est représentatif de ces nouvelles dynamiques mêlant objectifs internes et externes, investissements fonciers et développement agricole. Ce pays sahélien connaît une forte irrégularité interannuelle des productions agricoles pluviales. Sa population, en forte croissance, a des besoins alimentaires d'autant plus élevés que l'urbanisation se développe et que son niveau de vie augmente. Nourrir chaque année la population à partir des productions nationales est une priorité, car le pays dispose de peu de recettes 
d'exportation. Or, l'agriculture pluviale seule ne peut le permettre. Le Mali dispose en abondance de deux ressources clés pour la production agricole : la terre et l'eau (du fleuve Niger). Le gouvernement souhaite donc développer l'agriculture irriguée pour augmenter et sécuriser les productions.

La zone Office du Niger (ON) est un espace irrigué, essentiellement mis en valeur par des exploitations agricoles familiales. Historiquement, les terres aménagées, propriété éminente de l'État ${ }^{2}$, ont été principalement attribuées à des petits agriculteurs. En près de quatre-vingts ans, environ 100000 ha ont été aménagés et sont cultivés par 300000 paysans. Ces exploitations agricoles familiales assurent la majorité de la production rizicole nationale ${ }^{3}$. Plus récemment, de grandes entreprises se sont aussi installées, sur près de 7000 ha, essentiellement pour la production sucrière.

Dans un contexte de diminution de l'aide publique au développement, l'État malien n'a plus les moyens d'effectuer lui-même les investissements dans les infrastructures hydrauliques et l'aménagement de nouveaux casiers irrigués ${ }^{4}$. Pour pallier ce manque de capitaux, il opte pour le recours à des investisseurs. Il s'est ainsi engagé dans divers projets d'investissements, portés par des États étrangers ou des opérateurs privés ${ }^{5}$. L'État leur attribue des terres irrigables, au fort potentiel de production agricole; en contrepartie, ces investisseurs réalisent les aménagements nécessaires à l'irrigation et les exploitent.

5 L'arrivée d'acteurs économiques étrangers répond à une demande du gouvernement malien : produire pour alimenter les villes et consolider les filières agricoles. Mais les avantages de ces investissements sont moins évidents pour les exploitations familiales, dont les droits fonciers ne sont sécurisés ni sur leurs parcelles irriguées qui diminuent de génération en génération du fait de la croissance démographique, ni sur les terres exondées destinées aux cultures sèches, à l'élevage et au prélèvement de bois énergie ; ces terres sont en effet pour l'État, réputées "libres et sans maître " ${ }^{6}$. L'accès aux ressources pour les uns, les investisseurs, va évidemment peser sur les possibilités d'accès des autres, les exploitations familiales.

6 Une première partie de l'article présente le développement agricole actuel en zone $\mathrm{ON}$, mêlant agriculture familiale et agriculture entrepreneuriale.

7 La seconde partie de l'article analyse l'impact d'un projet d'agrobusiness sucrier sur les populations locales. Elle décrit la conception puis la mise en œuvre du Projet sucrier de Markala (PSM) et analyse les relations entre secteur privé et paysans, en partant des réactions de ces derniers à l'installation des investisseurs.

8 La conclusion porte sur les conséquences du développement des investissements sur les sociétés agraires de la zone $\mathrm{ON}$, la création de disparités sociales, le rôle du gouvernement et les limites apparentes des outils actuels de régulation de ces investissements.

\section{Du modèle paysan à l'agrobusiness}

\section{Les contraintes de la production agricole : l'accès aux ressources foncières et hydriques}

9 Dans la zone de l'office du Niger, 98000 ha bruts sont aménagés (chiffres de 2008); 7000 ha sont mis en valeur par des grandes entreprises, notamment en canne à sucre ; 
les 27000 exploitations agricoles familiales se répartissent sur 88000 ha, surtout pour la riziculture de saison et le maraîchage de contre-saison ${ }^{7}$.

Les exploitations paysannes sont soumises à des cahiers des charges contraignants (entretien du réseau, mise en valeur agricole, objectifs de rendement). Elles peuvent être évincées chaque année si la redevance en eau n'est pas payée. Les contrats d'exploitation annuels dont elles disposent ne leur donnent pratiquement aucune sécurité foncière.

11 Un autre aspect de cette insécurité foncière est la diminution, entre 1978 et 2003, des surfaces moyennes exploitées, de 7,5 à 2,4 ha par famille ${ }^{8}$. Croissance démographique et migrations en sont responsables. La pression foncière ne cesse de s'accroître.

Parce qu'il n'est plus en mesure de dégager les capitaux nécessaires à l'aménagement de nouveaux périmètres, l'État fait appel à des investisseurs pour développer le potentiel d'irrigation. On voit là le paradoxe de l'agriculture familiale, qui ne peut investir dans de nouveaux aménagements faute de fonds, et ne peut susciter des investissements publics devenus rares à son profit, alors qu'elle a fait la preuve, depuis vingt ans, de sa capacité à innover et à produire du riz pour le pays et des richesses pour la région.

13 Dans les discours officiels, les deux modèles, entrepreneurial et familial, ne sont pas en concurrence : moins de $10 \%$ des terres irrigables sont aménagées en zone $\mathrm{ON}$; il y a de la place pour tous et les terres ne seront pas retirées aux paysans. Grâce à certains projets, les terres disponibles pour les exploitations familiales seront même augmentées. Mais ce discours ne prend pas en compte le fait que les ressources en eau du fleuve Niger sont plus limitées qu'elles ne paraissent: si elles peuvent garantir l'irrigation de très importantes surfaces en période de crue, en période d'étiage, en revanche, il n'y a pas assez d'eau pour irriguer toute la superficie déjà aménagée. La concurrence va donc exister en contre-saison, où tous développent la riziculture et le maraîchage ou bien installent, pour les entreprises, des cultures pérennes à forte consommation en eau tout au long de l'année (canne à sucre, agrocarburants).

Même s'il n'y a pas de concurrence affichée, l'eau et les terres attribuées aux entreprises rurales ne le seront pas aux exploitations familiales. D'où l'importance des choix faits en matière de régulation des investissements privés.

\section{Investissements privés : entre offre et demande}

Les terres de la zone ON sont gérées par l'office du Niger, une entreprise publique qui peut octroyer à des investisseurs des baux pouvant atteindre quatre-vingt-dix-neuf ans pour des terres irrigables à aménager.

2005, afin d'attirer les investisseurs, l'État a actualisé le Code des investissements (loi 91-048/AN-RM du 26 février 1991 modifiée par la loi 05-050 du 19 août 2005) qui instaure un régime douanier et fiscal privilégié pour promouvoir les investissements de capitaux privés, nationaux et étrangers, dans les activités de production et de prestation de services. Il offre des avantages fiscaux, comme des exonérations d'impôts sur les bénéfices pouvant aller jusqu'à huit ans d'activité.

17 L'appel aux investissements privés a créé une forte dynamique. Sur la période 2004-2009 (avec une accélération à partir de 2007), 840 demandes ont été enregistrées 
et 870000 ha ont fait l'objet de demandes de bail, soit neuf fois la superficie des terres actuellement aménagées ${ }^{9}$.

Une agence d'accueil des investisseurs a été ouverte à Bamako par le gouvernement malien pour centraliser les demandes d'information et assurer la transparence de la procédure. Mais, dans la pratique, cette agence est peu consultée ; seuls s'y adressent les nouveaux agro-entrepreneurs de moindre taille, n'ayant aucun contact dans le pays. Le plus souvent, les investisseurs contactent directement des ministères, comme ceux de l'industrie ou de l'agriculture, voire la présidence de la République ${ }^{10}$. Ainsi, les projets sucriers négocient-ils directement avec le ministère de l'Industrie, arguant des avantages du développement des usines de transformation du sucre.

Ces relations directes illustrent le pouvoir d'influence de certains investisseurs et le poids de leurs arguments financiers et politiques. Cela leur permet de voir leurs demandes satisfaites en dehors des procédures officielles. Les modalités d'accès au foncier et à l'eau sont donc très inégales entre investisseurs et exploitants familiaux : alors que les premiers obtiennent en quelques mois des attributions provisoires sur des milliers d'hectares et bénéficient d'un accès à l'eau privilégié, les exploitations familiales peinent à obtenir quelques hectares et sont soumises en permanence à la menace de l'éviction.

20 Comme pour les terres, la dotation en eau est du ressort de l'office du Niger. Mais, là aussi, les investisseurs négocient les droits d'accès à l'eau ailleurs, le plus souvent directement avec des services de l'État central. L'ON ne sait d'ailleurs pas comment il pourra satisfaire tous les engagements qui ont déjà été pris... et qui de plus ne sont pas vraiment connus, une partie des accords n'étant pas rendus publics.

21 Les négociations pour le partage des terres et de l'eau sont donc déséquilibrées et échappent aux acteurs de terrain et aux gestionnaires officiels des deux ressources. Analysons ce processus pour le secteur de la canne à sucre et voyons les conséquences qui en découlent.

\section{Le développement de la canne à sucre : enjeux et réalités}

Actuellement, La canne à sucre est cultivée sur 7000 ha, et transformée sur place. Une entreprise chinoise, Sukala SA,gère le périmètre et les sucreries de l'ancienne entreprise d'État, créée au moment de l'indépendance et par la suite progressivement privatisée.

23 La production de la société Sukalane dépasse pas 35000 tonnes et le Mali a dû importer 139000 tonnes de sucre en 2009.

24 Ces chiffres expliquent que le secteur sucrier apparaît comme étant idéal pour le développement d'une agriculture irriguée moderne : fort potentiel agronomique pour la canne irriguée, expérience avérée de la culture, filière agro-industrielle intégrée et marché important à satisfaire.

Les demandes d'attribution de terres faites au gouvernement malien pour des projets sucriers portent sur plus de 60000 ha, dont 22000 ha d'extension demandés par Sukala. Trois projets sucriers sont prévus dans la zone de M’Bewani, à proximité du barrage de Markala sur le fleuve Niger. Le plus important, le Projet sucrier de Markala (PSM), entre dans le cadre d'un partenariat public-privé (PPP) entre le gouvernement malien et le 
groupe sud-africain à capitaux majoritairement britanniques Illovo, premier producteur africain de sucre.

L'état d'avancement des projets sucriers est très variable. Sur les 60000 ha ayant fait l'objet d'une demande foncière, entre 1998 et aujourd'hui, seuls 7000 ha sont effectivement mis en valeur; 300 ha sont toujours à l'état de tests agricoles. Certains projets sont en phase de négociation avec le gouvernement malien depuis plus de cinq ans. D'autres sont toujours au stade de «demande»: aucune étude ni projet définitif n'a été validé. Moins de 10000 ha sont aujourd'hui officiellement attribués.

Le projet PSM (Projet Sucrier de Markala) est emblématique de cette situation où l'ambition côtoie la confusion.

\section{Le projet PSM, mirage ou développement ?}

\section{Conception et mise en œuvre difficile}

Ce projet prévoit 40000 ha de canne à sucre et sa transformation industrielle. L'objectif est la production de $190000 \mathrm{t}$ de sucre blanc par an, avec des sous-produits importants : 15 millions de litres d'éthanol et 30 mégawatts d'électricité. Il a été initié, en 2002, par la Société sucrière de Markala (Sosumar). Dans son format actuel, sur les 14000 ha de la première tranche, $60 \%$ seraient exploités en régie (sur le modèle de l'entreprise chinoise Sukaladéjà en place) et $40 \%$ seraient exploités par des petits producteurs sous contrat.

30 À terme, l'activité économique générée par le projet devrait, selon l'investisseur, permettre de créer au moins 5000 emplois directs et 20000 emplois indirects. Le projet apporterait aussi près de 300000 euros et d'autres avantages pour la région (réalisation de pistes, investissements sociaux...).

31 Les porteurs de ce projet ont, dans un premier temps, recherché un partenaire privé pour son volet industriel. En 2005, un accord avec les groupes Illovo (Afrique du Sud) et Schaeffer (États-Unis) a été signé. Mais, depuis 2002, faute de financements, le projet peine à se concrétiser. Peu de réalisations sont visibles sur le terrain. Or, le démarrage des travaux était prévu pour 2007 et l'usine devait broyer ses premières cannes en décembre $2009^{11}$. En 2001, 1500 ha auraient déjà dû être en culture, dont 300 ha en riz pour nourrir les populations locales. En réalité, aujourd'hui, 140 ha de canne sont cultivés et aucune parcelle de riz ou de maraîchage n'a été aménagée. Seuls cinq employés permanents travaillent au sein du projet, ce chiffre est loin des 5000 emplois annoncés...

Les responsables sont toujours en négociation avec la BAD (Banque africaine de développement) pour trouver les fonds nécessaires au projet. Ils sont aussi continuellement en négociation avec le gouvernement malien pour obtenir de nouveaux avantages. Les promoteurs annoncent d'importantes disponibilités financières pour convaincre le gouvernement d'accorder de nouvelles facilités, présentées comme nécessaires pour la rentabilité du projet et l'obtention de financements.

La société Sosumar, qui porte le PSM, a déjà reçu en « cession directe à titre onéreux en toute propriété », une superficie de 857 ha, « libre de toute inscription, hypothèque ou autre droit », devant servir à l'implantation des installations industrielles. Les terres 
agricoles s'étendent sur plus de 20000 ha. Elles doivent être immatriculées et bornées pour que le bail emphytéotique soit reconnu. Les travaux topographiques indispensables sont considérables et n'ont toujours pas été réalisés.

La société Sosumar bénéficie aussi d'une convention signée avec le ministère de l'Industrie et du Commerce, qui lui accorde un débit de $20 \mathrm{~m}^{3} / \mathrm{s}$ pour couvrir les besoins en eau d'irrigation du projet. Pour l'ensemble de la superficie, un forfait de 3 millions d'euros paierait l'eau pour les cinquante ans du bail, soit moins de 3 euros par hectare et par an. Le rapport de 2009 déclare: "Aujourd'hui, il n'y a pas de problème de disponibilité en eau d'irrigation pour satisfaire les besoins en contre-saison de l'ON. » Néanmoins, une meilleure efficience de l'utilisation de l'eau est recherchée grâce aux pivots. En effet, une étude, réalisée par la $\mathrm{KfW}$ (Kreditanstalt für Wierderaufbau) en 2008, conclut que le maintien du débit minimum de $40 \mathrm{~m}^{3} / \mathrm{s}$ en aval de Markala n'est plus réaliste après l'aménagement d'un projet sucrier. En 2008 et 2009, le débit a été inférieur à ce niveau pendant soixante jours, alors que le PSM n'exploitait que 140 ha en tests ${ }^{12}$. La démonstration de la rareté de l'eau est faite par ce rapport. Si la convention garantissant un débit de $20 \mathrm{~m}^{3} / \mathrm{s}$ à la Sosumar est appliquée, comment pourront être satisfaits les besoins en eau des périmètres rizicole déjà existants?

La question des compensations est un autre point en suspens. Les mesures de compensation destinées aux populations locales ne sont pas clairement définies. Aucune n'est prévue pour les éleveurs nomades et transhumants qui font pâturer leurs troupeaux dans la zone depuis des siècles. Les rapports fournis par le promoteur ne précisent ni les effets du projet sur les droits d'usage des populations ni les conditions foncières d'accès aux terres du projet. Par contre, il est bien précisé qu'aucune contestation n'est juridiquement valable en ce qui concerne les droits fonciers que l'État malien va céder au projet à partir d'un titre foncier du domaine privé de l'État. Le promoteur deviendra, en fonction de l'avancée des négociations, propriétaire ou bailleurs des terres. Les droits coutumiers n'étant pas reconnus, les populations seront déplacées.

Selon le rapport prévoyant l'indemnisation des populations, en 2009, le PSM entraînerait le déplacement de près de 7000 personnes et la réinstallation de 1644 habitants. Les difficultés pour les populations sont essentiellement liées à la mise en place des pivots et à la création de grands champs de canne à sucre sur des terres traditionnellement utilisées pour la culture du karité (Vitellaria paradoxa). Le coût des indemnisations était estimé à près de 40 millions de dollars (aménagement de champs, dédommagement en espèces).

Face à cela, les paysans locaux ont tenté de manifester leur mécontentement.

\section{Réactions des paysans locaux et adaptations}

Comme d'autres projets d'investissements en zone $\mathrm{ON}^{13}$, ce projet a rencontré des problèmes d'acceptation par les populations de la zone. Six communes rurales sont touchées par sa mise en œuvre. Les paysans se sont manifestés à la suite de la signature de la convention, en 2005. L'un d'eux témoigne:

«On parle du sucre dans la zone, depuis 2002, des délégations sont venues voir les terres, mais depuis rien. Personne n'est venu dans les villages pour nous dire ce qui allait se passer pour nos champs. » 

projet réalise une nouvelle étude d'impact environnemental et social. Le gouverneur et les responsables du projet ont organisé, avec les responsables communaux, une consultation des populations locales. Les différentes attentes paysannes ont ainsi, selon le responsable du volet agricole, « été entendues » (construction d'écoles, de centres de santé...). Mais les réunions n'ont été tenues que dans deux villages, et de nombreux paysans, de hameaux ou villages éloignés, se sentent exclus.

Le projet a accepté d'aménager des terres en riz pour répondre aux revendications paysannes. Les familles reconnues comme devant être déplacées recevront en compensation des parcelles sur un périmètre rizicole de 1000 ha aménagé par le PSM. Afin d'intégrer certains paysans, le projet a également revu ses objectifs, pour y inclure une composante " agriculture contractuelle », en plus du mode d'exploitation en régie initialement prévu. La société en charge du périmètre sucrier, la Caneco, cultivera donc $60 \%$ des terres du périmètre en régie, et $40 \%$, soit 5600 ha, seront donnés en culture à des exploitants indépendants. Ces exploitants cultiveront de la canne à sucre et seront liés sous contrat à la Sosumar, l'antenne industrielle du PSM. Quelque 1250 ha, correspondant aux zones entre les pivots, seront mis en valeur à travers des cultures maraîchères, laissées en totalité à ces producteurs indépendants ; les 4350 ha restants seront obligatoirement cultivés en canne à sucre. Les modalités du contrat seraient les suivantes : chaque paysan aura l'obligation de cultiver de la canne à sucre; il pourra choisir sa parcelle ; le promoteur fournira la terre, les boutures de canne, les intrants, l'eau, le conseil et le suivi; l'agriculteur familial assurera la production et aura l'obligation de fournir l'ensemble de sa récolte de canne à la Sosumar. Les prix seront fixés en fonction du marché, pour le moment les cahiers des charges ne sont pas définis, mais ces producteurs indépendants devront avant tout répondre aux exigences de l'entité industrielle.

L'exigence d'un itinéraire technique contrôlé fait que la culture sera dirigée par les investisseurs. Le responsable du volet technique du projet annonce lors d'un entretien, en 2010 :

« Nous suivrons la culture technique, le paysan viendra ensuite vendre la canne à l'usine. Nous devons suivre la culture pour obtenir les résultats attendus. Les paysans, eux, feront ce qu'ils veulent sur les terres entre les pivots. Ils pourront faire du riz ou du maraîchage. »

(agriculteurs seront " surveillés » pour produire en quantité et en qualité la canne à sucre nécessaire à l'usine. Ils ne pourront cultiver, à leur gré, qu'entre les pivots, là où la maîtrise de l'eau est difficile et où circuleront les camions pour la livraison de la canne à sucre à l'usine.

Les paysans seraient "attributaires des parcelles", mais leurs droits fonciers exacts n'ont pas pu être identifiés; en cas de refus de cultiver la canne à sucre, les terres ne seraient pas attribuées. Le modèle, également mis en place au Sénégal et au Cameroun, reproduit en fait un modèle de régie d'État mécanisée, associée à des paysans en

Transcontinentales, 10/11 | 2011 
situation de "quasi-salariat sans garantie de salaire ${ }^{14}$, dont s'est progressivement libérée l'agriculture familiale rizicole.

Le choix d'inclure de l'agriculture contractuelle dans le PSM ne résulte, en fait, que du souci de mieux faire accepter le projet, après le conflit avec les populations locales. Ces annonces visent à calmer les paysans, mais ne sont pas pensées pour favoriser leur développement.

Afin de convaincre les paysans et d'éviter de nouveaux "débordements », le projet a aussi financé un voyage en Zambie pour six producteurs, issus des six communes concernées. La région visitée était celle de Mazabuka, où le groupe Illovo a mis en place un système similaire à celui envisagé à l'ON. Interrogés, en juillet 2010, les producteurs semblaient convaincus: «Les paysans là-bas ont une école et un centre de santé par village, les familles sont dans des logements autour de l'usine, et les producteurs reçoivent un appui technique et les intrants par l'usine qui leur rachète la canne. »

De retour de Zambie, les paysans pensaient qu'en quatre ans, ils pourraient se trouver dans les mêmes conditions que les paysans zambiens. Mais en Zambie, le groupe Illovo n'a pas établi le projet. Il a racheté des usines existantes, avec des plantations en place, déjà en phase de production: les quatre ans n'ont été que le temps nécessaire pour réaliser les infrastructures sociales. Au Mali, le projet n'a toujours pas réellement débuté. Les villageois réclament maintenant un nouveau voyage, pour quinze personnes, afin de pouvoir observer les réalités de la mise en œuvre sur le terrain d'un projet sucrier de cette ampleur. Mais les deux projets sont très différents par leur histoire, par leur contexte et par leurs infrastructures. Le projet du groupe, en Zambie, ne semble donc pas pouvoir être un modèle pertinent pour les paysans maliens, mais plutôt un instrument de communication destiné à leur donner confiance.

\section{Conclusion}

De nombreux projets sucriers basés sur de grandes exploitations intensives ont connu un certain succès. La culture irriguée de la canne à sucre à Richard-Toll, au Sénégal, permet de couvrir les deux tiers des besoins du pays et vise à l'autosuffisance pour 2013-2014 ${ }^{15}$. Mais ces grands projets ont également eu de lourdes conséquences sur les populations locales : diminution des cultures vivrières, non reconnaissance des droits antérieurs, création de disparités au sein des exploitations, contrat d'exploitation nonéquitable, endettement ${ }^{16} \ldots$

50 La logique de recherche du profit des grands projets risque d'accentuer ce type préjudices pour les populations locales de la zone ON. Les modalités de mise en œuvre des projets, le contenu des contrats, les droits et devoirs auxquels les paysans devront faire face, sont rarement connus des populations locales... et ne le sont même pas toujours des services de l'État. Les projets prennent forme à la suite de négociations politiques et économiques avec l'État hôte - ou plutôt certains de ses représentants/ services - sans que soit réellement fixé et rendu public le projet agricole. Les enjeux politiques et financiers dominent les enjeux techniques, économiques et sociaux; ils rendent aléatoires ces modèles de grandes exploitations intensives et menacent la durabilité des projets et leur adéquation avec les besoins des populations locales.

51 En fait, se pose la question de la régulation et de l'encadrement de ces investissements fonciers à grande échelle : les cadres juridiques et institutionnels des États hôtes sont 
généralement bafoués, souvent sans réaction de l'État qui tient lui-même un rôle moteur dans la négociation d'exceptions à ces cadres, puisque son objectif est de motiver l'investissement. Quels modes de régulation publique pourrait-on imaginer pour ces négociations asymétriques entre investisseurs favorisés et paysans marginalisés? Comment éviter le processus d'appauvrissement des exploitations familiales? Ces questions ne se posent pas qu'au Mali, on les retrouve, par exemple, à Madagascar ${ }^{17}$. Les institutions internationales comme la Banque mondiale ou la FAO, tentent de réguler les investissements fonciers à grande échelle en proposant des codes de bonne conduite ${ }^{18}$. Mais la mise en œuvre des cadres de régulation proposés dépend de la bonne volonté des investisseurs : les principes énoncés ne sont pas contraignants. Les dispositions relatives aux études environnementales et sociales développées par la Banque mondiale relèvent, elles aussi, du conseil aux investisseurs, mais aucune obligation ne les lie.

Les régulations d'ordre international sont faibles, voire inexistantes. La mise en application des projets est souvent très éloignée de leurs principes visant à respecter les droits des populations locales. La seule issue pour les paysans semble finalement être la revendication. Mais les différentes manifestations locales qui ont eu lieu dans la zone de Markala montrent que leur efficacité reste limitée: après huit ans de négociation, le projet PSM est toujours présent sans qu'aucun détail (technique de culture, contenu des contrats, rémunération, alimentation des marchés locaux) ne soit connu. Le poids de la société civile reste réduit, les populations sont largement dépassées par des accords économiques et politiques opaques négociés à un très haut niveau. À court terme, leur seul recours pourrait être de tenter de peser sur leurs représentants politiques, ce que permet le cadre politique démocratique malien.

\section{BIBLIOGRAPHIE}

COTULA L. (ed), « Land and water rights in the Sahel. Tenure challenges of improving access to water for agriculture », issue paper139, IIED (International Institute for Environment and Development), 2006.

DE JANVRY A., et al. (eds), Access to land, Rural Poverty and public actions, Oxford, Clarendon Press, 2001.

TOULMIN C., et J. QUAN, Evolving land rights. Policy and Tenure in Africa, London, DFID (Department for International Development), IIED and NRI (Natural Resources Institute), 2000.

\section{Publication récente des auteurs :}

A. Adamczewski, T. Hertzog, M. Dosso, P. Jouve et J.-Y. Jamin, « L'irrigation peut-elle se substituer aux cultures de décrue ? La dépression du lac Horo (Nord-Mali) », Cahiers Agricultures, vol. 20, $\mathrm{n}^{\circ}$ 1-2, 2011 : 97-104. DOI : 10.1684/agr.2011.0469.

J.-Y. Jamin, S. Bouarfa, J.-C. Poussin et P. Garin, « Les agricultures irriguées face à de nouveaux défis », Cahiers Agricultures, vol. 20, n 1-2, 2011 : 10-15. DOI : 10.1684/agr.2011.0477. 
B. Barbier, H. Ouedraogo, Y. Dembélé, H. Yacouba, B. Barry et J.-Y. Jamin, « L'agriculture irriguée dans le Sahel ouest-africain », Cahiers Agricultures, vol. 20, n 1-2, 2011 : 24-33. DOI : 10.1684/agr. 2011.0475.

J.-P. Tonneau et E. da Rocha Barros, « Agriculture irriguée dans la moyenne vallée du São Francisco : l'agriculture familiale cherche sa voie », Géoconfluences [en ligne], dossier " Le Brésil, ferme du monde », 2010, consulté en octobre 2011. URL : http://geoconfluences.ens-lsh.fr/doc/ etpays/Bresil/BresilDoc9.htm

J.-P. Tonneau et E. Sabourin, « Agriculture familiale et politiques publiques de développement territorial : le cas du Brésil de Lula ", Confins [en ligne], n 5, 2009, consulté en octobre 2011. URL : http://confins.revues.org/document5575.html

\section{NOTES}

1. L. Cotula, S. Vermeulen, R. Leonard and J. Keeley, Land grab or development opportunity? London/Rome, IIED (International Institute for Environment and Development), FAO (Food and Agriculture Organization) and IFAD (International Fund for Agriculture Development), 2009 ; J. von Braun, R. Meinzen-Dick, « "Land Grabbing” by Foreign Investors in Developing Countries: Risks and Opportunities ", IFPRI (International Food Policy Research Institute), Policy Brief, $\mathrm{n}^{\circ} 13$, April 2009 (Washington DC) ; FAO, IFAD, UNCTAD (CNUCED) and World Bank Group, « Principles for Responsible Agricultural Investment that Respects Rights, Livelihoods and Resources. Extended Version ", discussion note, 2010.

2. J.-Y. Jamin et M.-J. Doucet, «La question foncière dans les périmètres irrigués de l'Office du Niger (Mali) », Les Cahiers de la recherche-développement, $\mathrm{n}^{\circ}$ 38, 1994 : 65-82.

3. P. Bonneval, M. Kuper et J.-P. Tonneau (éd.), L'Office du Niger, grenier à riz du Mali: succès économiques, transitions culturelles et politiques de développement, Montpellier, Cirad/Karthala, 2002.

4. H. Turral, M. Svendsen and J.-M. Faures, «Investing in irrigation: reviewing the past and looking to the future ", Agricultural Water Management, $n^{\circ} 97,2010: 551-560$.

5. L. Cotula et al., op. cit.; GTZ, A. Diallo, G. Mushinzimana, A. Wehrmann, A. Üllenberg, «Foreign Direct Land Investment in Mali », Work of Division 45 - Agriculture, fisheries and food, GTZ, Eschborn, 2010.

6. Ordonnance $\mathrm{n}^{\circ}$ 00-027/P-RM du 22 mars 2000 portant Code domanial et foncier au Mali.

7. Y.-M. Coulibaly, J.-F. Bélières et Y. Koné, « Les exploitations agricoles familiales du périmètre irrigué de l'Office du Niger au Mali : évolutions et perspectives. », Cahiers Agricultures, vol.15, nº 6, $2006: 562-569$.

8. J.-F. Bélières, T. Hilhorst, D. Kébé, M. S. Keïta, S. Keïta et O. Sanogo, « Irrigation et pauvreté : le cas de l'office du Niger au Mali. », Cahiers Agricultures [en ligne], vol. 20, n 1-2, 2011 : 144-149, consulté en octobre 2011. URL : http://dx.doi.org/10.1684/agr.2011.0473

9. SEDIZON, Situation des attributions de terres en bail dans la zone Office du Niger, Bamako, SEDIZON, Mai 2010.

10. A. Adamczewski, P. Burnod, H. Papazian, Y. Coulibaly, J.-Y. Jamin, J.-P. Tonneau, Investments in Irrigable Land for Large-Scale Agricultural Land In Mali. Conference Africa for Sale: Analysing and Theorizing Foreign Land Claims and Acquisitions, 28-29 October 2010, Groningen (Netherlands), Groningen University, 2010.

11. "Le sucre de Markala sous contrôle sud-africain », Jeune Afrique [en ligne], 12 juin 2006, consulté en octobre 2011. URL : http://www.jeuneafrique.com/Article/LIN11066lesucniaciro/

12. R. Schüttrumpf et T. Bökkers, Analyse du potentiel d'irrigation lors de la saison sèche dans la zone de l'Office du Niger, Rapport final, Adama Sangare, GTZ, 2008. 
13. F. Brondeau, "L'agrobusiness à l'assaut des terres irriguées de l'office du Niger (Mali)», Cahiers Agricultures [en ligne], vol. 20, n 1-2, 2011 : 136-43, consulté en octobre 2011. URL : http:// dx.doi.org/10.1684/agr.2011.0472

14. R. Hirsch, Étude économique du casier rizicole de Richard-Toll (S.D.R.S.), 1953-1971. Étude hydroagricole du bassin du fleuve Sénégal, Saint-Louis (Sénégal), FAO/OERS, 1972.

15. Voir le site web de la Compagnie sucrière sénégalaise : http://www.css.sn

16. J.-L. Chaléard, «Les mutations de l'agriculture commerciale en Afrique de l'Ouest », Annales de géographie, $\mathrm{n}^{\circ}$ 596, 1996 : 563-583.

17. A. Teyssier, A. Ramarojohn et R. Andrianirina Ratsialonana, « Des terres pour l'agro-industrie internationale? Un dilemme pour la politique foncière malgache ", EchoGéo [en ligne], $\mathrm{n}^{\circ} 11$, 2010, consulté en octobre 2011. URL : http://echogeo.revues.org/11649

18. World Bank, «Large scale acquisition of land rights for agricultural or natural resourcebased use ", concept note, 18 February 2009 ; id., " Rising global interest in farmland. Can it yield sustainable and equitable benefits?», report, September 2010 ; O. De Schutter, « Large-scale land acquisitions and leases: a set of core principles and measures to address the human rights challenge », UNO, 11 June 2009.

\section{RÉSUMÉS}

Le phénomène d'appropriation foncière à grande échelle touche, depuis plusieurs années, le continent africain. Soucieux de moderniser leur agriculture et de trouver des capitaux pour la développer, de nombreux États ont fait le choix de recourir à des investisseurs privés étrangers. $\mathrm{Au}$ Mali, dans le plus grand périmètre irrigué d'Afrique de l'Ouest, des milliers d'hectares sont maintenant attribués à des investisseurs. Le Projet sucrier de Markala (PSM), issu d'un partenariat entre le gouvernement malien et un investisseur privé sud-africain, porte sur près de 40000 hectares. Fort de nombreuses expériences en Zambie et en Afrique du Sud, premier producteur de sucre du continent, l'investisseur souhaite reproduire au Mali son modèle industriel : culture de canne à sucre en régie et avec des paysans sous contrat. Le projet entre en concurrence directe avec des paysans maliens pour l'accès à la terre et à l'eau. Il a en effet deux répercussions majeures : l'exclusion des paysans des parcelles et une compétition pour l'accès à l'eau. À partir des réactions paysannes, l'article analyse la cohabitation entre exploitations agricoles familiales et nouveaux investisseurs, ainsi que les disparités locales qui se créent. Alors que le gouvernement souhaite développer l'agrobusiness, l'accompagnement et la régulation des investissements sont peu effectifs.

For several years, the African continent has been affected by large-scale land appropriations. Many governments have resorted to foreign private investors to modernise their agricultural sectors and raise capital for agricultural development. In Mali, which has the largest irrigated sector in Western Africa, investors now own thousands of hectares. The Markala Sugar Project (MSP), a partnership between the government and a private South African investor, covers some 40,000 hectares. Following initiatives in Zambia and South Africa (the continent's largest sugar producer), the investor hopes to reproduce a similar business model in Mali: sugar cane production by the owner and under contract. The project competes directly with Malian farmers for land and water in two ways. It excludes Malian farmers from plots of land and competes with them for access to water. Using farmers' reactions, the article analyses the cohabitation between 
family farms and new investors, as well as the local disparities that arise. At a time when the government hopes to further develop agribusinesses, investment regulation mechanisms remain ineffective.

\section{INDEX}

Mots-clés : périmètre irrigué, développement agricole, accaparement foncier, investisseurs privés, paysans

Keywords : irrigated sector, agricultural development, accaparement foncier, private investors, paysans

\section{AUTEURS}

\section{AMANDINE ADAMCZEWSKI}

Elle est doctorante en géographie au CIRAD (Centre de coopération internationale en recherche agronomique pour le développement) de Montpellier, inscrite à l'école doctorale ED 60, «Territoire, temps, sociétés et développement » de l'université Montpellier-III. Ingénieur agronome, spécialisée en gestion sociale de l'eau, elle a débuté sa thèse en 2010. Son travail de recherche porte sur la gestion foncière des terres irrigables en zone Office du Niger au Mali, les dynamiques d'investissements, les arrangements fonciers et les logiques d'acteurs.

CIRAD UMR G-Eau, TA C-90/15, 73, rue Jean-François Breton, 34398 Montpellier Cedex 5 Tél. : + $33(0) 467166487$

amandine.adamczewski@cirad.fr

\section{JEAN-YVES JAMIN}

Agronome, chercheur au CIRAD de Montpellier, il travaille au sein de l'UMR G-Eau (Gestion de l'eau, acteurs, usages) sur les usages agricoles de l'eau. Ses travaux portent sur l'évolution des systèmes de culture et des systèmes de production dans les périmètres irrigués et sur les relations entre l'eau, l'agriculture et les acteurs agricoles et non-agricoles de la gestion de l'eau. Ses terrains de travail sont l'Afrique de l'Ouest, Madagascar, le Brésil et l'Asie du Sud-Est.

CIRAD UMR G-Eau, TA C-90/15, 73, rue Jean-François Breton, 34398 Montpellier Cedex 5 Tél. : + 33 (0)4 67166485

jamin@cirad.fr

\section{JEAN-PHILIPPE TONNEAU}

Agronome et géographe, il est directeur de l'UMR Tetis (Territoires, environnement, télédétection et information spatiale) au CIRAD. L'activité principale de Tetis porte sur l'utilisation de l'information spatiale (en particulier les images satellitaires) par le monde rural. Son activité de recherche est consacrée à la conception, l'exécution et le suivi de projets de recherche en appui au développement durable territorial. Dans ce cadre, il développe des activités de recherche, d'expertise et de formation sur l'organisation spatiale des territoires, les modèles de développement, les politiques publiques et l'élaboration de plans et de schémas régionaux d'aménagement. Il a 
longuement travaillé en Afrique (Guinée-Bissau, Burkina Faso) et au Brésil. Il a participé à des opérations de recherche dans près de trente pays du Sud.

Maison de la Télédétection, 500, rue Jean-François Breton, TA C-91 / MTD (Bât. M.

Téléd., Bur. 105), 34398 Montpellier Cedex 5

Tél. : + 33 (0)4 67548715

jean-philippe.tonneau@cirad.fr 